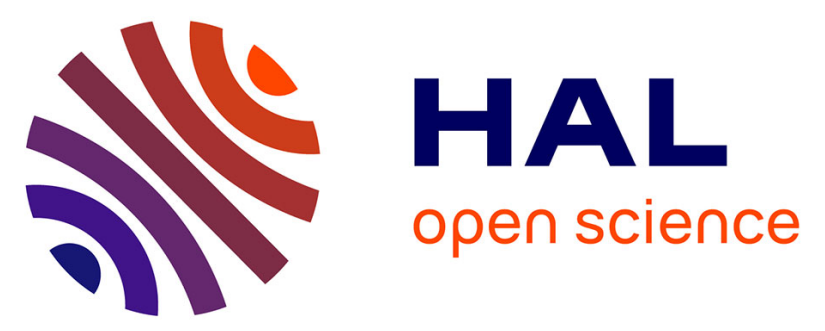

\title{
Integration of electronic effects into molecular dynamics simulations of collision cascades in silicon from first-principles calculations
}

Thomas Jarrin, Nicolas Richard, Johannes Teunissen, Fabiana da Pieve, Anne Hémeryck

\section{To cite this version:}

Thomas Jarrin, Nicolas Richard, Johannes Teunissen, Fabiana da Pieve, Anne Hémeryck. Integration of electronic effects into molecular dynamics simulations of collision cascades in silicon from first-principles calculations. Physical Review B, 2021, 104 (19), pp.195203. 10.1103/PhysRevB.104.195203 . hal-03455747

\author{
HAL Id: hal-03455747 \\ https://hal.laas.fr/hal-03455747
}

Submitted on 29 Nov 2021

HAL is a multi-disciplinary open access archive for the deposit and dissemination of scientific research documents, whether they are published or not. The documents may come from teaching and research institutions in France or abroad, or from public or private research centers.
L'archive ouverte pluridisciplinaire HAL, est destinée au dépôt et à la diffusion de documents scientifiques de niveau recherche, publiés ou non, émanant des établissements d'enseignement et de recherche français ou étrangers, des laboratoires publics ou privés. 


\title{
Integration of electronic effects into molecular dynamics simulations of collision cascades in silicon from first-principles calculations
}

\author{
Thomas Jarrin and Nicolas Richard* \\ CEA, DAM, DIF, F-91297, Arpajon, France \\ Johannes Teunissen and Fabiana Da Pieve \\ Royal Belgian Institute for Space Aeronomy BIRA-IASB, 1180, Brussels, Belgium \\ Anne Hémeryck ${ }^{\dagger}$ \\ LAAS-CNRS, Université de Toulouse, CNRS, Toulouse, France
}

\begin{abstract}
The inclusion of sophisticated density-dependent electronic stopping and electron-phonon coupling calculated with first-principles methods into molecular dynamics simulations of collision cascades has recently become possible thanks to the development of the so-called EPH (for Electron-PHonon) model. This work aims at employing the EPH model in molecular dynamics simulations of collision cascades in Si. In this context, the electronic stopping power is investigated in Si at low energies with Ehrenfest Dynamics calculations. Also, the parametrization of the EPH model for Si, from firstprinciples Ehrenfest Dynamics simulations to actual molecular dynamics simulations of collision cascades, is performed and detailed. We demonstrate that the EPH model is able to reproduce very closely the density-dependent features of the energy lost to electrons obtained with ab initio calculations. Molecular dynamics collision cascade simulations results obtained in Si using the EPH model and the simpler but widely employed Two Temperature Model (TTM) are compared, showing important discrepancies in the collision cascades results obtained depending on the model employed.
\end{abstract}

\section{INTRODUCTION}

Accurate Molecular Dynamics (MD) simulations of collision cascades into semiconducting materials are of high interest for applications in hazardous radiation environments such as space [1] and nuclear power facilities [2], where sensitive microelectronic and optoelectronic devices like bipolar junction transistors and image sensors are subjected to intense fluxes of energetic particles. Neglecting the non-Coulomb interactions occurring at very high energies only (on the order of $\mathrm{MeV}$ ), energetic charged particles into matter transmit their kinetic energy through nuclear stopping and electronic stopping: when the energies at stake are very high, electronic stopping is by far the leading loss mechanism, whereas when the energies are low, nuclear stopping becomes the dominant mechanism. In a collision cascade event, defects are created as a result of Coulomb interactions between the atoms set in motion and those at rest, i.e. nuclear stopping. Electronic stopping, which describes the excitation of electrons of the target material by the projectile, is not directly responsible for the creation of defects in collision cascades. However, an accurate evaluation of the energy going into atomic displacements, and thus a complete model of collision cascades, must take into account the energy lost to electrons. Indeed, the latter can have important implications on the dynamics of the ions and the recombination of the defects. The well-known Lindhard-Scharff-Schiott (LSS) theory is an example of

\footnotetext{
* nicolas.richard@cea.fr

$\dagger$ anne.hemeryck@laas.fr
}

successful attempts to develop a unified model for electronic and nuclear stopping [3, 4]. Nevertheless, this theory does not allow for a dynamic atomic-scale treatment of collision cascades. Thus, for many years, MD simulations could not take into account electronic stopping but only nuclear stopping via repulsive empirical potentials, the best-known being the Moliere potentials [5] and the ZBL potentials [6]. Fortunately, in the last two decades, several models accounting for electronic stopping and/or electronic-phonon coupling have been developed to be used in addition to MD simulations, which are inherently able to correctly account for nuclear stopping through the interatomic potential.

The simplest form of these models accounts for electronic stopping, via a friction force, but does not account for electron-phonon coupling [7]. Another form, probably the most employed one, is the derivation of the Two Temperature Model (TTM), developed by Duffy and Rutherford [8] for MD simulations from earlier works by Caro and Victoria [9]. This model accounts for both electronphonon coupling and electronic stopping in the framework of a Langevin thermostat [10]: a friction force acts as the electronic stopping and a stochastic force term embodies the electron-phonon coupling. Despite the major breakthrough that this model represented, it suffers from certain limitations. Firstly, the electronic density is considered constant in the entire model The electronic stopping power thus cannot be crystal direction-dependent as it was confirmed to be by recent experiments [11] and Time Dependent Density Functional Theory (TDDFT) calculations [12]. Secondly, a recent paper pointed out [13] the large uncertainties in the choice of values for some parameters of the model, due to the lack of the- 
oretical or experimental data in the literature. In response to the limitations raised above, a model, named EPH (for Electron-PHonon) [14, 15], has been developed recently, taking as a basis the framework of a Langevin thermostat, such as the TTM, but incorporating the notion of spatial locality in the model through the possibility to define a non constant electronic density in the simulation box [14]. In the EPH model, the electronphonon coupling is seen as an electronic stopping process, which allows the construction of a unified model for ion-electron interactions. Since the electronic density is not constant in the model, values of the electronic stopping power with respect to the electronic density must be provided as input to the simulation. To obtain it, it is necessary to resort to Ehrenfest Dynamics (ED) calculations of electronic stopping power. The ED framework can go beyond linear response and Real-Time TDDFT (RT-TDDFT) by propagating both the dynamics of the electrons with RT-TDDFT and the dynamics of the nuclei via integration of the classical equations of motions of the nuclei. In the following, the acronym TDDFT is used to refer to ED calculations as it is commonly done in the literature of electronic stopping calculations. As TDDFT has been shown to correctly describe the high energy electronic stopping power regime [16], but also electron-phonon processes occurring at very low energies (typically meV) [17], the EPH model therefore provides a framework for MD simulations accounting for electronic effects with very high accuracy. The model and its use on $\mathrm{Ni}$ and $\mathrm{Ni}$ alloys is described in [15] and [18].

The purpose of the present paper is to parametrize the $\mathrm{EPH}$ model for a semiconductor, silicon $\mathrm{Si}$, with TDDFT calculations. We aim to demonstrate its ability to reproduce electronic stopping calculated by TDDFT within MD simulations and we compare the results of MD collision cascades in Si with different EPH and TTM parametrizations. Section II describes the EPH model and gives the computational details of our TDDFT and MD simulations. Section III is dedicated to TDDFT calculations of electronic stopping in Si for different energies and crystal directions. In Section IV is detailed how to parametrize the EPH model. The results of the collision cascades in Si obtained with the EPH model and the TTM are compared and discussed in Section V.

\section{METHODS}

In this section, the EPH model is described and the methodologies as well as computational details employed in TDDFT and MD simulations are given.

\section{A. EPH model}

The EPH model is currently implemented as a fix plug-in for LAMMPS [19]. It was developed and implemented by A. Tamm and A.A. Correa. It is based on the Langevin framework they derived in [14] where a system of ions exchanges energy with a bath of electrons of temperature $T_{e}$. In this framework, the scalar values of friction and random forces of usual Langevin models (among them the TTM), acting as the electronic stopping and the electron-phonon coupling in the TTM respectively, are replaced by many-body tensor notations, allowing an accurate description of electronic stopping and electron-phonon coupling processes under the same model. The usual MD equation of motion for particle $I$ therefore becomes:

$$
m_{I} \vec{a}_{I}=\vec{F}_{I}^{a d i a b}-\sum_{J} B_{I J} \vec{v}_{J}+\sum_{J} W_{I J} \vec{\xi}_{J}
$$

In the above equation, $m_{I}$ is the mass of particle $I, \vec{a}_{I}$ its acceleration, $\vec{F}_{I}^{a d i a b}$ the conservative forces (deriving from the interatomic potential in MD simulations), $\vec{v}_{I}$ its velocity, $\sum_{J} B_{I J} \vec{v}_{J}$ the friction force acting on particle $I$ where $B_{I J}$ are $I J$ components of the $B$ tensor defining the magnitude of the friction force and $\sum_{J} W_{I J} \vec{\xi}_{J}$ the random force acting on particle $I$ where $\vec{\xi}_{J}$ are uncorrelated Gaussian random variables normalized to $2 k_{B} T_{e}$ and $W_{I J}$ are $I J$ components of the $W$ tensor defining the magnitude of electron-phonon coupling. The $W$ and $B$ tensors are related via the following expression deriving from the fluctuation-dissipation theorem [20]:

$$
B_{I J}=\sum_{K} W_{I K} W_{J K}^{T}
$$

The magnitudes of both the electronic stopping power and the electron-phonon coupling entirely lie within the $W_{I J}$ terms. This choice made in the EPH model is based on the works of Caro et al. [9] and Koponen [21] from the early 90s, as well as on more recent results by Caro et al. [17], which assert that electronic stopping and electronphonon coupling are the "opposite limits of the same basic governing equations" (or mechanism) [21]. In other words, based on the same physics of energy exchange, the electronic stopping power describes the case of high velocity projectiles sampling regions of various electronic densities, while electron-phonon coupling corresponds to low velocity atoms oscillating around their equilibrium position of relatively low electronic densities. This aspect is made explicit by the correlations between friction and random forces shown in (2). The definition of $W$ must ensure the conservation of angular and linear momentum [14] in order to have a rich electron-phonon mode. To this end, the matrix terms $W_{I J}$ of the $W$ tensor are defined as follows in the MD-EPH model:

$$
W_{I J}= \begin{cases}-\alpha_{J}\left(\overline{\rho_{J}}\right) \frac{\rho_{I}\left(r_{I J}\right)}{\overline{\rho_{J}}} \vec{e}_{I J} \otimes \vec{e}_{I J} & (I \neq J) \\ \alpha_{I}\left(\overline{\rho_{I}}\right) \sum_{K \neq I} \frac{\rho_{K}\left(r_{I K}\right)}{\bar{\rho}_{I}} \vec{e}_{I K} \otimes \vec{e}_{I K} & (I=J)\end{cases}
$$


with $\rho_{I}\left(r_{I J}\right)$ the electronic density created by atom $I$ at a distance $r_{I J}, \bar{\rho}_{I}=\sum_{J \neq I} \rho_{J}\left(r_{I J}\right), \vec{e}_{I J}$ are unit vectors of the $I J$ direction and $\alpha_{I}\left(\overline{\rho_{I}}\right)$ parameters which control the friction and random forces magnitudes. This last term is the key parameter to be specified by the user. From the user's point of view, in the EPH model, electronic stopping power and electron-phonon coupling are defined by the same single $\alpha(\rho)$ parameter. In theory, the low $\rho$ part of $\alpha(\rho)$ coupled to the low velocities of the ions governs the electron-phonon coupling part. This is very different from the strategy adopted in the TTM framework, in which two distinct parameters must be defined for electronic stopping power and electron-phonon coupling. The EPH model was developed to reproduce TDDFT data on electronic stopping. Thus, the $\alpha$ parameters appearing in (3) must be optimized to fit TDDFT results. This aspect is the most difficult and most important part of parametrizing the EPH model, and this article describes how to do it for Si in Section IV. The whole TDDFT calculations and the process of fitting to TDDFT were already performed on $\mathrm{Ni}$ and $\mathrm{Ni}$ alloys in [15] and [18].

In the model, the electronic density is not the real one because it would require solving the Schrödinger equation at each timestep of the MD simulation. The electronic density is approximated by considering that the electronic density around each atom of the system is that of the atomic sphere density of the isolated atom. At a given point of the simulation box, the electronic density is thus the sum of the contributions of the atomic sphere electronic density of all the atoms located within a certain cutoff radius of this given location. The users of the EPH model must provide the densities of the atomic spheres of the species involved. This atomic sphere approximation is known to work well for metals [22] for which electrons are delocalized, but the more localized covalent bonds of semiconducting materials might decrease the accuracy of this approximation. We discuss this aspect in Section IV. In this work, the OPIUM code [23] is used to calculate the atomic sphere density of $\mathrm{Si}$.

\section{B. TDDFT calculations}

TDDFT calculations of electronic stopping are performed using the QB@LL code [24, 25], following the procedure detailed in [26]. All our simulations are performed on $3 \times 3 \times 3$ bulk diamond supercells of Si with the experimental lattice constant of $5.431 \AA$ [27]. Periodic boundary conditions are employed to accurately model a bulk system. Calculations are carried out with the PBE approximation for the exchange and correlation functional and a norm conserving pseudopotential with 4 valence electrons $\left(3 s^{2} 3 p^{2}\right)$ is used. The results obtained with this pseudopotential are compared in Section III with results we obtained with another pseudopotential with 12 valence electrons. This 12 valence electrons pseudopotential is adapted to work with PBE functionals from the one generated by Lee et al. in [12] with the OPIUM code [23]. Indeed, it has been vividly demonstrated in [16] and [28] that, at least at high energies, the core electrons of the target and of the projectile can be excited. We use a plane-wave cutoff of 100 Ry with the 4 valence electrons pseudopotential and a cutoff of 220 Ry with the 12 valence electrons pseudopotential.

The initial condition required for the real-time propagation is obtained by first performing a self-consistent calculation of the system consisting of the supercell and the projectile. Then, the electronic wavefunctions are propagated in time from the wavefunctions obtained from the static calculation, and the classical equations of motions of the nuclei are integrated in time, with force calculations derived from the electronic Hamiltonian and the electronic wavefunctions.

The velocity of the projectile is kept constant, and the forces on all atoms are set to zero. Thus, we only record, through the increase of the total energy, the contribution of electronic excitations. In this paper, we are only interested in neutral $\mathrm{Si}$ projectiles in bulk $\mathrm{Si}$ diamond at energies ranging from $10 \mathrm{keV}$ to $200 \mathrm{keV}$. These energies are typical of collision cascades events. Our goal is to have a statistical treatment of MD simulations of collision cascades, but with energies higher than a few tens of $\mathrm{keV}$, this is not possible because of the associated computational costs.

Below $50 \mathrm{keV}$, we use a timestep of 1 as, and from $50 \mathrm{keV}$ and above we employ a timestep of 0.5 as. The timesteps are chosen to ensure energy conservation, no influence on the calculations of the electronic stopping powers and reasonable computational cost.

We simulated the ions and electrons dynamics in the trajectories of $<001>,<110>$ and $<111>$ center and offcenter channels as well as in directions incommensurate with the crystal lattice. Incommensurate directions are chosen following the indications of [26], such that they avoid channeling directions and head-on collisions. All the directions cited above were used for the optimization of the $\alpha(\rho)$ function of the EPH model, but electronic stopping powers were only calculated on trajectories in the $\langle 001\rangle,\langle 110\rangle$ and $\langle 111\rangle$ directions. To extract the stopping power from the simulations in the channels, we calculated the slope of the linear regression of the total energy against the distance $x$ traveled by the projectile, but only between $x=1 / 2 \times a_{\text {lat }}$ and $x=5 / 2 \times a_{\text {lat }}$ for the $<001>$ direction, $x=\sqrt{2} / 2 \times a_{\text {lat }}$ and $x=5 \sqrt{2} / 2 \times a_{\text {lat }}$ for the $\langle 110\rangle$ direction, and $x=\sqrt{3} / 2 \times a_{\text {lat }}$ and $x=5 \sqrt{3} / 2 \times a_{\text {lat }}$ for the $<111>$ direction. Doing this, we ignore the short transient state at the beginning, and we completely eliminate the contribution of the lattice without having to resort to Born Oppenheimer Approximation-MD (BOA-MD) simulations. See [29] for more details on this. 


\section{MD simulations}

We use the LAMMPS code for MD simulations [30]. The cascades are initiated with $10 \mathrm{keV}$ PKAs (Primary Knock-on Atoms) in Si. Boxes of 1000000 atoms made of $50 \times 50 \times 50$ diamond-like unit cells and 4096000 atoms made of $80 \times 80 \times 80$ diamond-like unit cells are employed. The simulation boxes are divided into two areas: in the outer cells the velocities are rescaled to maintain the temperature at a desired value (thus absorb the thermal wave), and the inner cells form an NVE ensemble in which the atoms evolve freely to simulate the collision cascade.

The initialization of the simulation is done by scaling the velocities of all atoms, such that the overall temperature is the desired one ( $300 \mathrm{~K}$ in the current work). For calculations carried out with the TTM, the system is equilibrated for at least $20 \mathrm{ps}$ with a timestep of $1 \mathrm{fs}$. With the EPH, we found that this equilibration time needed to be increased to 100 ps. Since the velocity of the atoms drastically changes throughout the cascade, the integration timestep is changed during the simulations. We imposed the condition that no atom moves more than $0.02 \AA$ between two steps of the simulation, with a timestep varying between $0.001 \mathrm{fs}$ and $1 \mathrm{fs}$.

We employed the Stillinger-Weber (SW) potential developed in [31]. To better describe short interatomic distances, the SW potential is combined to a repulsive twobody potential. The chosen repulsive potential is the Ziegler Biersack Littmark potential (ZBL) [6]. SW and ZBL potentials are combined together through a Fermi function as in [32]:

$$
V_{t o t}(r)=(1-F(r)) V_{Z B L}(r)+F(r) V_{S W}(r)
$$

where $r$ is the distance between two atoms, $V_{t o t}$ is the total potential, $V_{Z B L}$ the repulsive ZBL potential, $V_{S W}$ the SW potential and $F$ the Fermi function used to link the two potentials. The expression of the Fermi function as well as its parameters values used for Si can be found in [33].

To correctly account for the stochasticity of the cascades, the distributions and statistical quantities are based on 75 simulations in distinct and independent directions [34]. We usually use sets of 100 simulations but the high computational cost of simulations with the EPH model compelled us to reduce this number to the already satisfactory one of 75 . The method employed to choose the directions is the "Symmetry" method detailed in [34].

Depending on the simulations performed, the electronic stopping TTM parameter $\left(\gamma_{s}\right)$ was optimized to best reproduce the TDDFT stopping data, or was derived from SRIM calculations [35]. Details of the TTM and $\mathrm{EPH}$ parameters employed are given when needed.

\section{ELECTRONIC STOPPING POWER CALCULATIONS}

In this section, we are interested in extracting the electronic stopping power from the evolution of the total energy with respect to the distance traveled by the projectile in TDDFT calculations, in center and off-center $(1 / 2)<001>$ channel trajectories for energies ranging from $10 \mathrm{keV}$ to $200 \mathrm{keV}$. By off-center $(1 / 2)$ we mean that the initial position of the projectile is $\left(1.125 \times a_{\text {lat }}\right.$, $\left.1.5 \times a_{\text {lat }}, 0.0\right)$. Trajectories along the $<110>$ and $<111>$ channels have only been investigated with $100 \mathrm{keV}$ projectiles.

The purpose of our TDDFT calculations is to allow parametrizing the EPH model for MD simulations of collision cascades. Thus, we are interested in low-energy projectiles, which explains why simulations are carried out with Si projectiles from $10 \mathrm{keV}$ to $200 \mathrm{keV}$. While for higher energies it has been proven to be mandatory, at least in $\mathrm{Ni}$ [16], to employ pseudopotentials with semicore electrons in the valence, no pseudopotential-dedicated study was performed for the low energies of interest for us. However, a detailed study with a 12 valence electrons pseudopotential has been performed for Si in [12].

Here, we ran some simulations to determine whether it is necessary to employ a pseudopotential with semicore electrons in the valence, which would have a considerably higher computational cost. For comparison, we performed a simulation in the $<001>$ center channel with a projectile of $700 \mathrm{keV}$ with both a 4 valence electrons pseudopotential and a 12 valence electrons pseudopotential. The pseudopotential with 4 valence electrons gives a stopping power of $33.9 \mathrm{eV} / \AA$, compared to $53.6 \mathrm{eV} / \AA$ with the pseudopotential with 12 valence electrons. The reported value with the pseudopotential with semicore electrons in the valence is in good agreement with the calculations performed with a similar pseudopotential in [12]. Making the same comparison for a $200 \mathrm{keV}$ projectile along the same trajectory, we find an electronic stopping of $16.7 \mathrm{eV} / \AA$ with the 4 valence electrons pseudopotential and of $16.0 \mathrm{eV} / \AA$ with the 12 valence electrons pseudopotential.

The lower value found with the pseudopotential explicitly integrating semicore electrons is counter-intuitive. It can be explained by the fact that the 12 valence electrons pseudopotential generated with OPIUM supports only one projector per angular moment, which means that it had to be generated for the reference configuration $2 s^{2} 2 p^{6} 3 s^{0} 3 p^{0}$ and not the neutral configuration $2 s^{2} 2 p^{6} 3 s^{2} 3 p^{2}$. As a result, it suffers from transferability problems which could easily explain uncertainties in the electronic stopping calculations. However, the results are similar enough to state that below $200 \mathrm{keV}$ a minima, a pseudopotential with more than 4 valence electrons is of no use. All the results presented in the following were obtained with the 4 valence electrons pseudopotential.

In Fig. 1, the electronic stopping powers of initially neutral Si projectiles into bulk Si along the center $<001>$ 
channel and the off-center $<001>$ channel $(1 / 2)$ are shown with respect to the velocity of the projectile. The results of TDDFT calculations from [36], experimental results [11] and SRIM results are also shown for comparison.

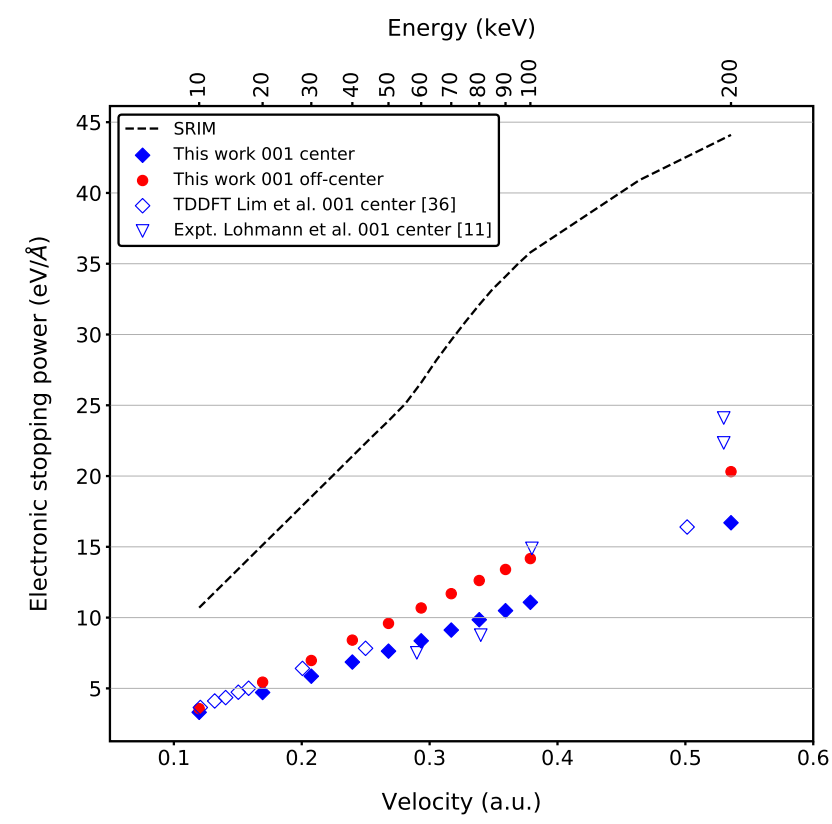

FIG. 1. Electronic stopping powers with respect to the velocity of the projectile in a.u. calculated for projectiles of energies ranging from $10 \mathrm{keV}$ to $200 \mathrm{keV}$ (solid markers) and compared to stoppings from other computational (empty diamond markers) and experimental (empty triangle markers) references and calculated with SRIM (dashed line).

According to Fig. 1, the increase in electronic stopping power in $\mathrm{Si}$ for projectiles between $10 \mathrm{keV}$ and $200 \mathrm{keV}$ in the $<001>$ channel is clearly linear with the projectile velocity. Our calculations compare really well with the results presented in [36]. A slight discrepancy can be noticed, which could not be explained neither by the use of LDA vs PBE, nor by the different lattice parameters employed, according to additional calculations we performed.

The stopping powers found for the off-center $<001>$ trajectories are a bit higher than those for the center, and this discrepancy increases with the projectile velocity. Our results for the center channel and those of [36] are in good agreement with the state-of-the-art experimental results of [11] for the $<001>$ center channel. This gives us confidence in the reliability of the electronic stopping powers calculated from TDDFT despite the fact that our stoppings are 2 to 3 times smaller than SRIM stopping powers. This last point is of course partly due to the fact that we only consider results for the $<001>$ channels, whereas the electronic stopping in SRIM is a kind of average over all possible directions. Some of the stoppings reported for the different sampled trajectories for the $100 \mathrm{keV}$ projectile in Table I are already closer to
TABLE I. Electronic stopping powers calculated with TDDFT for various directions for a neutral Si projectile of $100 \mathrm{keV}$.

\begin{tabular}{c|c} 
& Elec. stop. $(\mathrm{eV} / \AA)$ \\
\hline$<001>$ center channel & 11.1 \\
$<110>$ center channel & 9.8 \\
$<111>$ center channel & 13.8 \\
$<001>$ off-center channel $(1 / 2)$ & 14.2 \\
$<001>$ off-center channel $(1 / 4)$ & 19.5 \\
$<110>$ off-center channel & 12.2 \\
\hline SRIM & 35.8
\end{tabular}

the SRIM value.

The disagreement between SRIM and TDDFT stoppings for $\mathrm{Si}$ in $\mathrm{Si}$ at low energies has already been pointed out in [36]. More recent work by Lee et al. at higher energies in [12] reports TDDFT electronic stoppings for initially charged $\mathrm{Si}^{+12}$ projectiles up to twice greater than the SRIM stoppings, compared to TDDFT stoppings up to twice lower for neutral $\mathrm{Si}^{+0}$ projectiles, which is consistent with our results with neutral Si projectiles.

Trying to reproduce SRIM values of electronic stopping power requires running very long TDDFT simulations on various random trajectories (see [29]) and with various initial projectile charge states to obtain converged values of the stopping. Doing so is beyond the scope of this paper.

\section{PARAMETRIZATION OF THE EPH MODEL}

This section is dedicated to the parametrization of the parameter $\alpha(\rho)$ of the EPH model. Following (3), $\alpha$ is expressed in $\left(\mathrm{eV} \cdot \mathrm{ps} / \AA^{2}\right)^{1 / 2}$ (default units of the model). The parameter that we really have to adjust, according to [15], is homogeneous to (eV.ps $\left./ \AA^{2}\right)$, thus the square of $\alpha$. It is sometimes called $\beta$ in [15] and [18], has the same role in $B_{I J}$ as $\alpha$ has in $W_{I J}$, as can be understood from (3) and (2), and can be seen as the electronic stopping power of the projectile divided by its velocity. The original papers of the EPH model $[14,15,18]$ do not explicitly mention $\beta$ in the equations although they actually name it in the text and optimize it. Although we actually do optimize the $\beta$ parameter and not the $\alpha$ parameter, as $\alpha$ appears in the equations, the $\alpha$ parameter is the parameter discussed in the following.

For any set of distinct projectile trajectories, the best parametrization is the one that gives an evolution of the energy lost to the electrons as a function of the distance that is the closest to that obtained with TDDFT calculations. The energy lost to the electrons is a direct output of the EPH model, whereas it requires an additional step to be obtained from TDDFT calculations. To obtain only the energy transmitted to the electrons by the projectile ion, the contribution of the lattice to the total energy must be subtracted. In practice, the energy with 


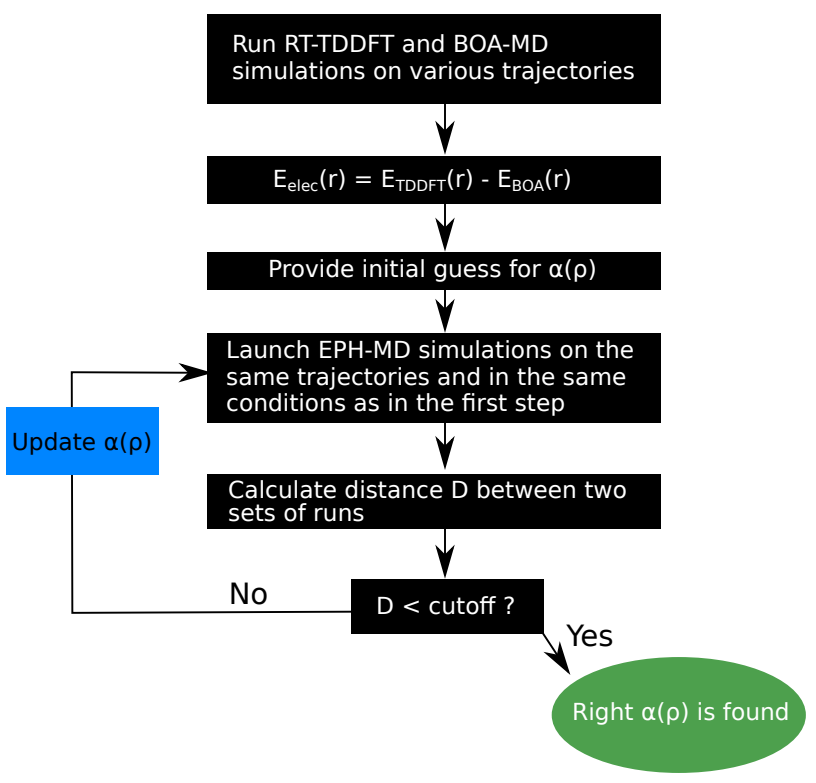

FIG. 2. Optimization scheme of the $\alpha(\rho)$ parameter.

respect to distance obtained with a $\mathrm{BOA}-\mathrm{MD}$ run on a given trajectory $E_{B O A}$ must be subtracted from the energy with respect to distance obtained with TDDFT on the same trajectory $E_{T D D F T}$. At a given distance $r$ on the projectile path, the energy $E_{\text {elec }}(r)$ lost to electrons in a TDDFT run thus is:

$$
E_{\text {elec }}(r)=E_{T D D F T}(r)-E_{B O A}(r)
$$

In this article, when an energy loss to electrons obtained with TDDFT is mentioned, it was calculated with (5). It doubles the number of simulations to be performed because for a single trajectory, one TDDFT simulation and one BOA-MD simulation are required. Since the parameterization of the EPH model requires to run many TDDFT calculations in different trajectories, it is computationally prohibitive to fit the model with simulations launched with too low velocities. Indeed, the lower the initial energy (velocity) of the projectile, the longer the simulation time. Thus, we fit the EPH model with TDDFT calculations initiated with $100 \mathrm{keV}$ projectiles, even if we will then perform our MD simulations of collision cascades with lower initial energies. By doing so, we reach an affordable computational cost. This approximation is reasonable considering that the EPH model assumes that the electronic stopping is linear with velocity, which is always the case for metals, for which the EPH model has been developed. In the case of Si, Lim et al. demonstrated in [36] that the electronic stopping is metal-like above $3 \mathrm{keV}$, which means that it is linear with the velocity.

Our calculations in the $<001>$ channel confirmed this observation (see Fig. 1) for projectiles from $10 \mathrm{keV}$ to $200 \mathrm{keV}$. Also according to the work of Lim et al., below $3 \mathrm{keV}$ and above $60 \mathrm{eV}$, the bandgap induces changes as electronic stopping power still increases with velocity but faster than above $3 \mathrm{keV}$. Below $60 \mathrm{eV}$, the stopping was found to be non-zero. However, at low energies (velocities) the contribution of electronic effects is less important than at high energies and the initial energies we use for MD simulations of collision cascades are well above $3 \mathrm{keV}$. Consequently, the errors coming from this linear stopping approximation should be small.

To rank the different MD-EPH (MD-TTM) parametrizations, an appropriate distance measure between the energy lost to electrons along a given projectile trajectory during the reference TDDFT simulations and the MD-EPH (MD-TTM) simulations must be defined. We use the Mean Absolute Error (MAE):

$$
M A E=\frac{1}{n} \sum_{i}^{n}\left|E_{M D}\left(r_{i}\right)-E_{T D D F T}\left(r_{i}\right)\right|
$$

where $n$ is the number of points sampled along the trajectory and $r_{i}$ is the distance traveled by the projectile associated to point $i . E_{M D}$ in this equation describes either the energy lost to electrons during a MD-EPH or a MD-TTM simulation.

An optimization scheme for the $\alpha(\rho)$ parameter of the EPH model can now be established. This scheme is presented in Fig. 2.

We fit the EPH model using ten different trajectories: $<001>$ center channel and two off-center trajectories, $<110>$ center channel and one off-center trajectory, $<111>$ center channel and also four incommensurate directions, one integrating a vacancy (one missing atom) on the projectile path. Table II summarizes the characteristics of all sampled directions.

The conditions of the MD-EPH runs are the same as for the TDDFT runs: the forces on all atoms are set to zero and the projectile moves with a constant velocity. For comparison, MD-TTM simulations in the same conditions are also run. In the TTM and EPH runs in this section, the electron-phonon coupling is switched off, so we only consider the electronic stopping. In the TTM, the total friction coefficient is actually the sum of the electronic stopping parameter $\gamma_{s}$ and the electronphonon coupling parameter $\gamma_{p}$ [8]. This means, in the TTM calculations, we neglect the energy exchanges between ions and electrons via the stochastic force term (electron-phonon coupling) and we only consider the parameter $\gamma_{s}$ in the friction term. Activating the electronphonon coupling would not change the results, but would make necessary an extra smoothing step of the energy lost to electrons curves.

It was found in [37] that the electronic stopping power is a multivalued function of the electronic density: for a single value of the electronic density there are several values of electronic stopping. The parameters $\alpha(\rho)$ cannot be a multivalued function of the density. Thus, the stopping retrieved from the EPH model can only approximate the reference TDDFT stopping. We want to make 
TABLE II. Main characteristics of the trajectories sampled for fitting the EPH model.

\begin{tabular}{l|c|c} 
& Initial projectile position & Velocity unit vector \\
\hline \hline Center channel $<001>$ & $\left(1.25 \times a_{\text {lat }}, 1.5 \times a_{\text {lat }}, 0.0\right)$ & $(0.0,0.0,1.0)$ \\
Center channel $<110>$ & $\left(0.0,0.0,1.625 \times a_{\text {lat }}\right)$ & $(1 / \sqrt{2}, 1 / \sqrt{2}, 0.0)$ \\
Center channel $<111>$ & $\left(1.25 \times a_{\text {lat }}, 1.25 \times a_{\text {lat }}, 0.0\right)$ & $(1 / \sqrt{3}, 1 / \sqrt{3}, 1 / \sqrt{3})$ \\
Off-center channel $<001>(1 / 2)$ & $\left(1.125 \times a_{\text {lat }}, 1.5 \times a_{\text {lat }}, 0.0\right)$ & $(0.0,0.0,1.0)$ \\
Off-center channel $<001>(1 / 4)$ & $\left(1.0625 \times a_{\text {lat }}, 1.5 \times a_{\text {lat }}, 0.0\right)$ & $(0.0,0.0,1.0)$ \\
Off-center channel $<110>$ & $\left(0.0,0.0,1.81 \times a_{\text {lat }}\right)$ & $(1 / \sqrt{2}, 1 / \sqrt{2}, 0.0)$ \\
Incommensurate 1 & $\left.1.25 \times a_{\text {lat }}, 1.5 \times a_{\text {lat }}, 0.0\right)$ & $(0.3620,0.0 .1134,0.9252)$ \\
Incommensurate 1 vacancy & $\left(1.25 \times a_{\text {lat }}, 1.5 \times a_{\text {lat }}, 0.0\right)$ & $(0.3620,0.0 .1134,0.9252)$ \\
Incommensurate 2 & $\left(1.25 \times a_{\text {lat }}, 1.5 \times a_{\text {lat }}, 0.0\right)$ & $(0.4017,0.1867,0.8965)$ \\
Incommensurate 3 & $\left(1.25 \times a_{\text {lat }}, 1.25 \times a_{\text {lat }}, 0.0\right)$ & $(0.1922,0.4364,0.8790)$
\end{tabular}

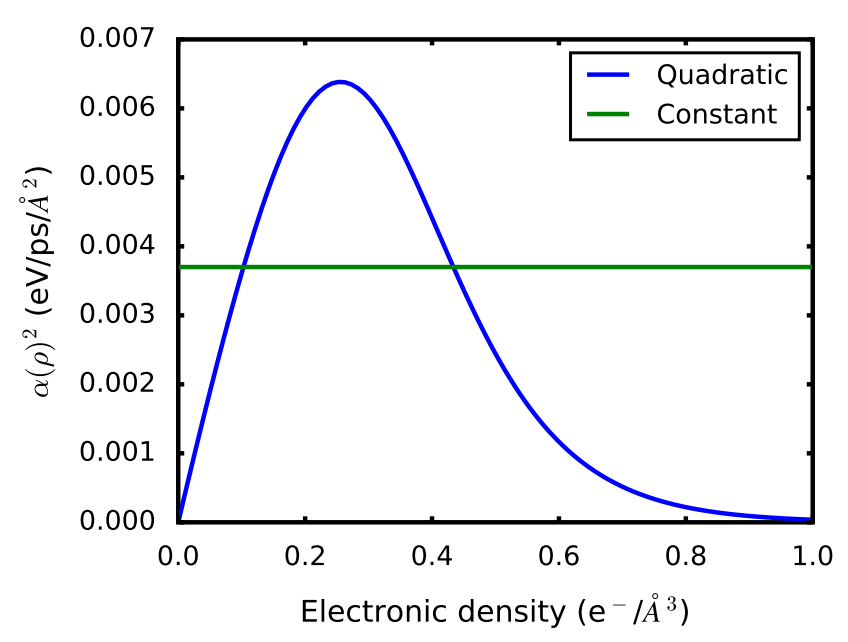

FIG. 3. Constant and quadratic optimized $\beta(\rho)=\alpha(\rho)^{2}$ functions.

it as true as possible to the TDDFT data using a singlevalued function for the parameter $\alpha$. In [15], Caro et al. used a complex optimization algorithm procedure with spline and knot points for $\alpha(\rho)$.

In order to demonstrate the accessibility of the EPH model, we chose to employ two simple analytical expressions for $\alpha(\rho)$ : one where $\alpha$ is kept constant, and another one where $\alpha(\rho)$ has a quadratic expression of the form $\left(a \rho+b \rho^{2}\right) \times\left(1+e^{c\left(\rho-\rho_{f}\right)}\right)^{-1}$. The exponential decay term in the quadratic expression must be added to the quadratic expression otherwise the stopping would be drastically overestimated at high $\rho$. This is also motivated by the observations made in [15] that the electronic stopping tends to saturate for very high values of the electronic density. The quadratic expression has no constant term in order to get it to be equal to 0 at $\rho=0$.

In the following, the data corresponding to the optimized $\alpha$ parameters are shown. The best optimization is defined when the total MAE, calculated from the sampled positions of all the studied trajectories, is the smallest. Fig. 3 represents the optimized $\alpha(\rho)$ parameters with respect to density in those two cases. The optimized expressions of the $\alpha$ parameter in the constant and quadratic cases respectively give:

$$
\begin{aligned}
& \text { - } \alpha(\rho)^{2}=0.0037 \mathrm{eV} / \mathrm{ps} / \AA^{2} \\
& \text { - } \alpha(\rho)^{2}=\left(0.041 \times \rho+0.0 \times \rho^{2}\right) \times \frac{1}{1+e^{10(\rho-0.3)}} \mathrm{eV} / \mathrm{ps} / \AA^{2}
\end{aligned}
$$

Starting from a quadratic expression of $\alpha(\rho)$, we obtained a linear one in the end with the $b$ parameter strictly equal to 0 . As the final linear expression we obtain originates from the optimization of an initially quadratic expression, the term quadratic will be kept in the following.

Fig. 4 and Fig. 5 show the energy lost to the electrons in channeling and incommensurate trajectories obtained with TDDFT, the EPH model and the TTM, respectively. Table III displays the MAE in the constant and quadratic EPH scenarios and the TTM fitted to TDDFT case only, for all the studied trajectories. Note that the TTM electronic stopping parameter in the TTM curves below named "TTM fitted" (see Fig. 4 and Fig. 5) was also optimized to minimize their MAE with respect to the TDDFT data. Contrarily, the TTM parameters of the simulations whose corresponding curves are entitled "TTM SRIM" are not fitted to TDDFT data. In this case, the electronic stopping power parameter $\gamma_{s}$ is obtained from the SRIM calculations of electronic stopping power.

The most blatant feature, common to all the graphs of Fig. 4, is the fact that the energy lost to electrons with the TTM in its SRIM-derived parametrization is far greater than the energy lost to electrons calculated with TDDFT. Considering the existing discrepancy we highlighted in Section III between our TDDFT electronic stopping results and the SRIM stopping calculations, this result was expected.

We also clearly see an increase in the slope of the energy lost to electrons when regions of higher densities are sampled. This is particularly clear in Fig. 4(e) and all the incommensurate directions of Fig. 5, in which the projectile encounters high values of electronic density on its path. However, at high values of electronic density, the slope of the energy lost to electrons stops increasing and stabilizes. The increase in electronic stopping thus seems to saturate when high values of electronic density are reached. Visually, from the figures just cited, 


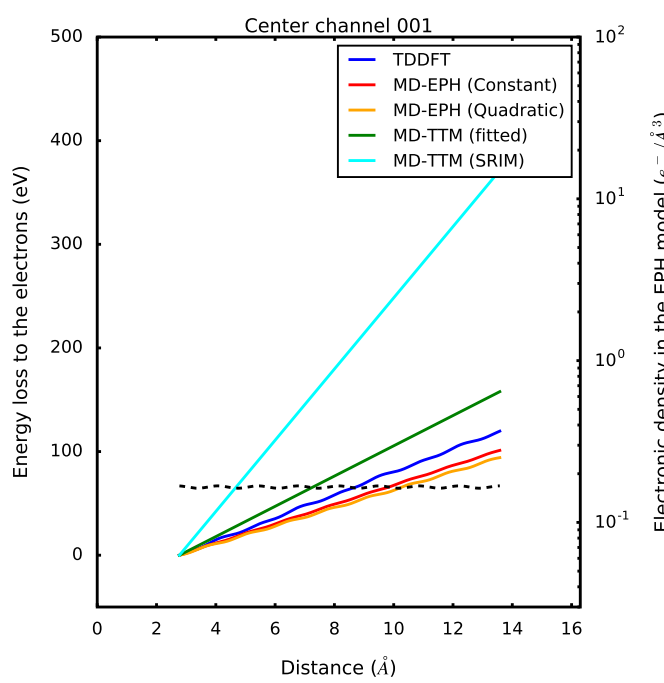

(a)

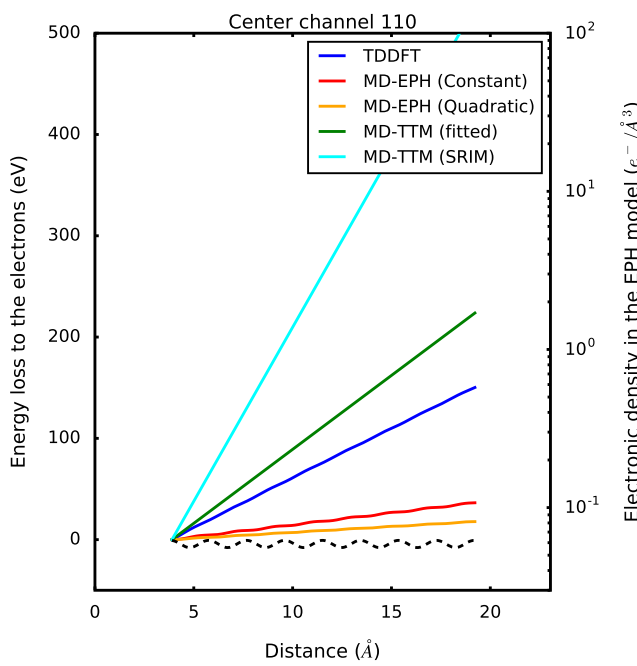

(b)
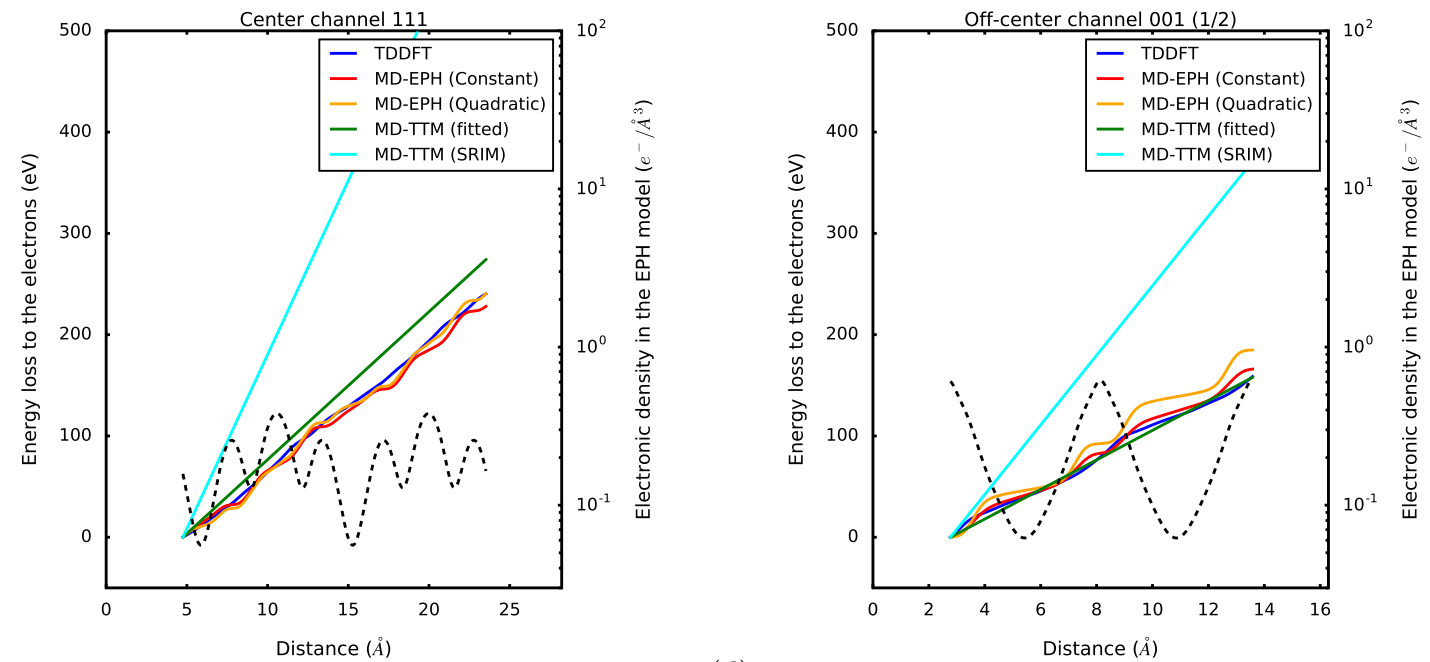

(c)

(d)
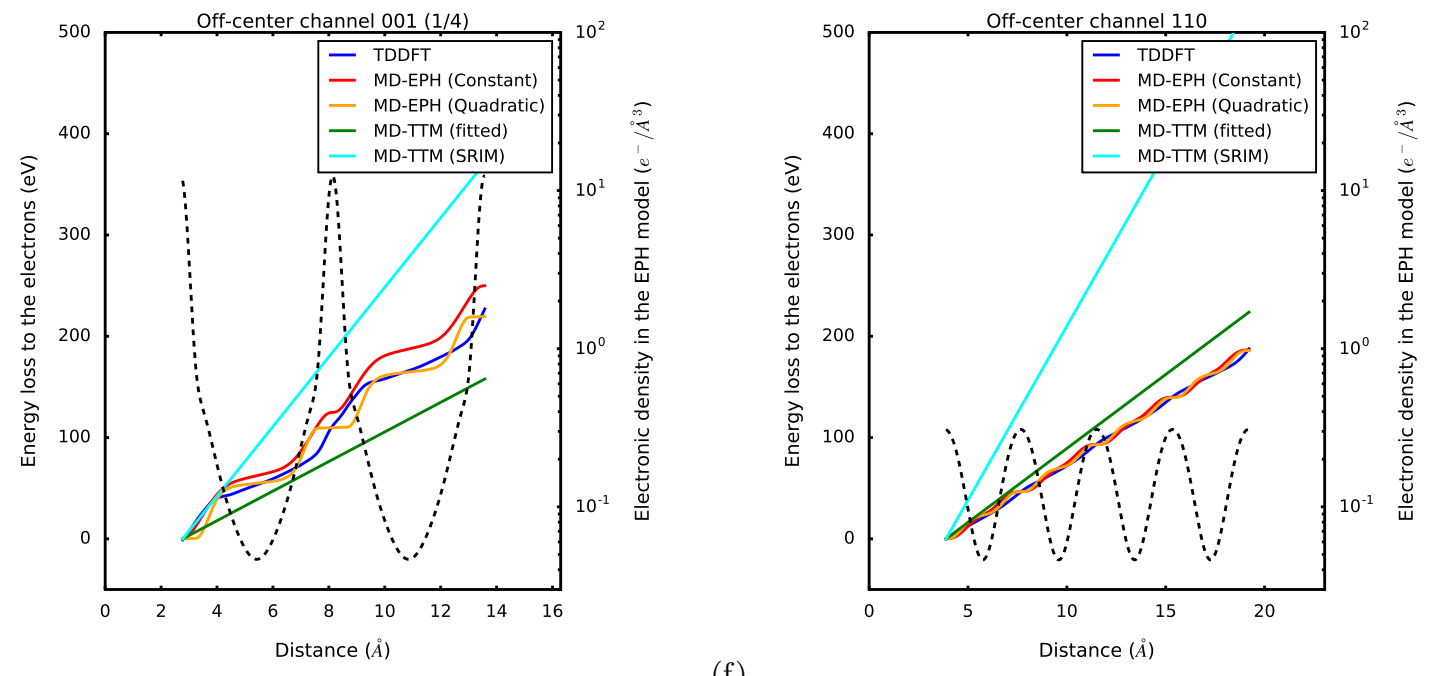

(e)

FIG. 4. Energy lost to the electrons by the projectile for all the channel trajectories studied with $100 \mathrm{keV}$ Si projectiles in Si calculated with TDDFT, the EPH model and the TTM (solid lines). The electronic density seen by the projectile in the EPH model is represented in each case with a dashed black line. We define the zero energy reference for all the curves at the x-axis point at which we start the fitting. The curves are plotted on the same symmetric portions of the trajectories as the ones we defined in Section II B for the calculations of stopping powers. Beware that the right y-axis for the electronic density are in log-scale, and that the $\mathrm{x}$-axis are not the same in each graph. 


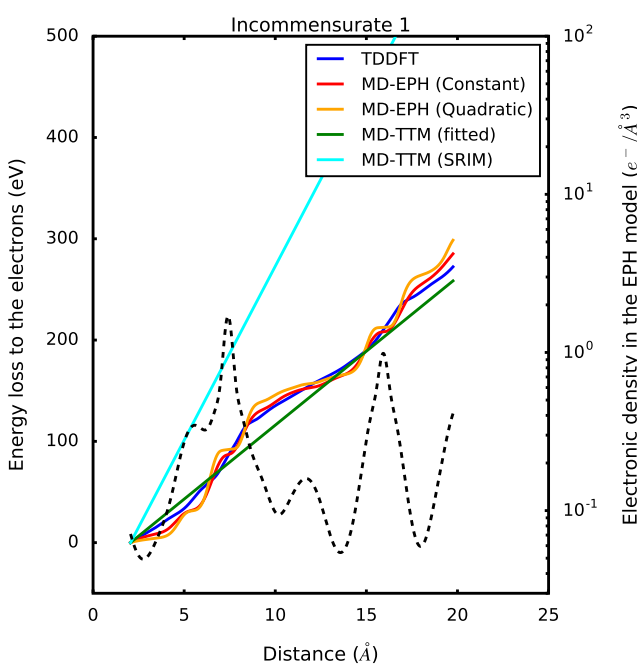

(a)

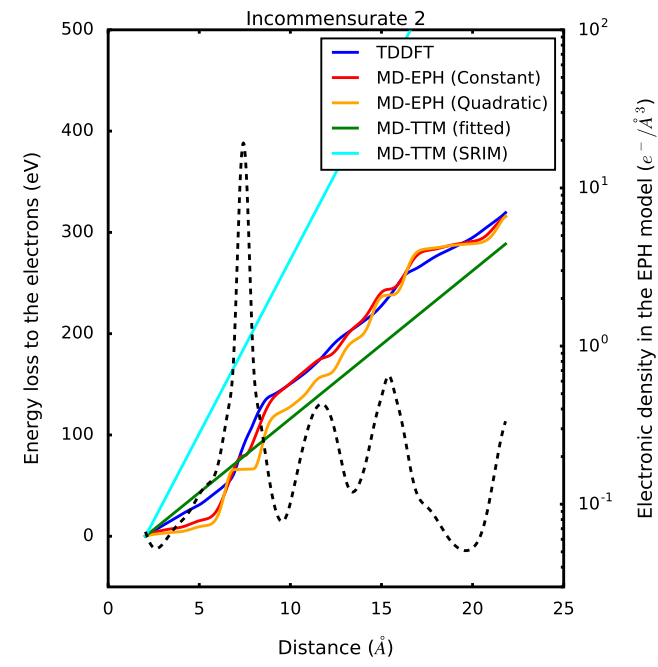

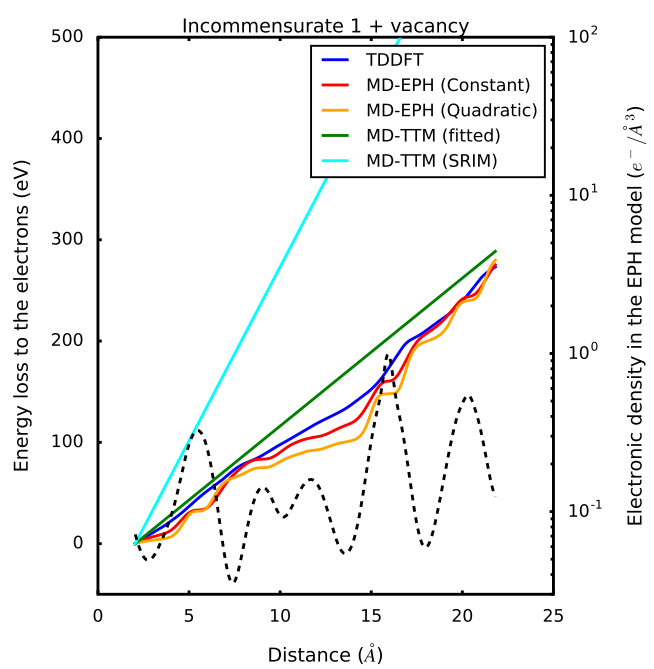

(b)

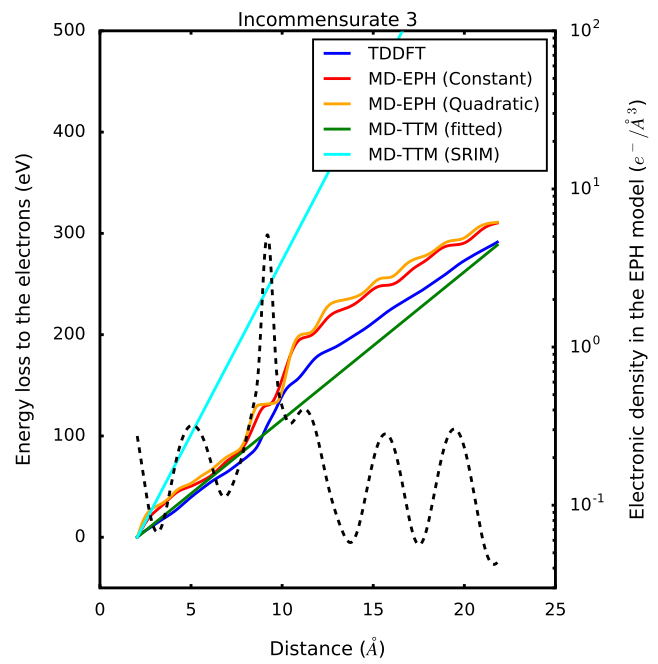

(d)

FIG. 5. Energy lost to the electrons by the projectile for all the incommensurate trajectories studied with $100 \mathrm{keV}$ Si projectiles in Si calculated with TDDFT, the EPH model and the TTM (solid lines). The electronic density seen by the projectile in the EPH model is represented in each case with a dashed black line. We define the zero energy reference for all the curves at the $\mathrm{x}$-axis point at which we start the fitting. Beware that the right $\mathrm{y}$-axis for the electronic density is in log-scale.

the EPH model also provides an increase in the stopping power when the electronic density increases, just as in the TDDFT results. This can be observed in Fig. 4(d), Fig. 4(e), Fig. 4(f) as well as in all the graphs of Fig. 5, in which the changes in the slopes of the energy lost to electrons with changes in the electronic density are obvious. This constitutes a first qualitative satisfactory aspect of the EPH model.

We now focus on the channel trajectories of Fig. 4. The TDDFT trajectories of the $<001>$ off-center channel $(1 / 2),<110>$ off-center channel and $<111>$ center channel of Fig. 4 are very well reproduced by the constant parametrization of the EPH model, the density dependence of the energy lost to electrons being followed very closely by MD-EPH simulations. This is confirmed by Table III and the low MAE associated to these trajectories. The quadratic parametrization also reproduces quite well the $<110>$ off-center channel and $<111>$ center channel, but does not perform as good as the constant case for the $<001\rangle$ off-center channel $(1 / 2)$ : we get a MAE of $4.2 \mathrm{eV}$ for the constant case compared to $13.4 \mathrm{eV}$ in the quadratic case.

The $<001>$ center channel and $<001>$ off-center channel $(1 / 4)$ do not follow the reference TDDFT data curves as closely as the previously cited trajectories but still are satisfactory. The constant $\alpha$ function provides better results for the $<001>$ center channel (MAE of $9.4 \mathrm{eV}$ compared to $12.9 \mathrm{eV})$, but the situation is reversed for the $<001>$ off-center channel (1/4) (MAE of $15.5 \mathrm{eV}$ compared to $8.0 \mathrm{eV})$. 
Following the good results obtained with the EPH model for the five trajectories already mentioned, it is surprising that for the $\langle 110\rangle$ center channel trajectory, the MD-EPH simulations are far below the reference TDDFT data (Fig. 4(b)). The origin of this large discrepancy might be due to the fact that the electronic density approximated by the EPH model for this trajectory might be very low compared to the actual electronic density that the projectile sees in the TDDFT simulation. However, we compared the EPH and $a b$ initio electronic densities calculated with Quantum Espresso [38] for various trajectories including the center $<110\rangle$. We found that in all cases, the densities are similar. Therefore, it cannot explain the discrepancies we observe for the center $<110>$ channel between EPH and TDDFT.

Choosing a different analytic expression $\alpha(\rho)$ would certainly help to obtain a better agreement for the case of the $\langle 110\rangle$ channel, but it could also mean working with more complex and possibly physically meaningless expressions. A possibility, which is not to be omitted to explain the observed discrepancies, is that the $<110>$ center channel emphasizes a non-linear aspect of electronic stopping the EPH model is unable to capture. With the EPH model, at low electronic densities, the electronic stopping power is roughly proportional to the electronic density. Moving from Fig. 4(b) to Fig. 4(a), we see that the electronic density is multiplied by a factor of about 4-5, and that the same is observed for the energy lost to electrons with the EPH model. However, the energy lost to electrons obtained with TDDFT is nearly unchanged between the $<001>$ center channel and $<110>$ center channel (in agreement with the results of Table I), whereas we observed that the ab initio electronic density is very close to that displayed in Fig. 4(a) and Fig. 4(b). Further work should be conducted to be conclusive on this point.

In Fig. 4(e) one can notice a step-like behavior of the energy lost to electrons in the EPH model. This behavior is intrinsic to the model, but arises for lower values of the electronic density in the quadratic parametrization of the EPH model. Indeed, the parameter $\alpha(\rho)$ in the quadratic parametrization smoothly decreases to zero. It means that when $\rho$ exceeds the value for which $\alpha(\rho)=0$, no energy is transferred between ions and electrons and that the energy lost to electrons levels off until the projectile reaches a lower value of $\rho$.

Although the TTM fitted energy loss curves in the channel cases of Fig. 4 are linear, they do not perform that bad in many cases, as can be observed in Fig. 4. According to Table III, the MAE is even smaller in the TTM fitted case than in both MD-EPH scenarios for the $<110\rangle$ center channel, and smaller than the quadratic case for the $<001>$ center channel $(1 / 2)$ : MAE of $3.6 \mathrm{eV}$ with the TTM fitted compared to $13.4 \mathrm{eV}$ in the quadratic case. However, the TTM fitted performs clearly worse than the constant and quadratic EPH cases for the $<111>$ center channel, $<001>$ off-center channel $(1 / 4)$ and $<110>$ off-center channel trajectories.
Looking at Fig. 4(e), it is obvious that the worst agreement between TTM fitted and TDDFT is obtained for the $<001>$ off-center channel $(1 / 4)$. Since the electronic density reaches very high values in this case, the TTM fitted largely underestimates the value of the stopping since it cannot vary with the electronic density contrary to the stopping in the EPH model.

Now focusing on the incommensurate directions of Fig. 5, the TTM curves in their SRIM-derived parametrizations show again very large discrepancies with the reference TDDFT data.

We also observe with the incommensurate directions that during close encounters (collisions) between the projectile and an atom of the lattice, the energy lost to electrons in the EPH model in the plots of Fig. 5 is step-like. This degrades the similarities between the TDDFT and the EPH data. Due to this step-like behavior, rather than searching for point-by-point correspondence between the TDDFT data and our EPH curves during those close encounters, we aim to obtain a good fit between EPH and TDDFT data on average during the collision. In other words, we aim to have the value of energy lost to electrons with the EPH model as close as possible to the TDDFT data, after the collision.

Despite this step-like behavior, visually from Fig. 5, the comparison of different incommensurate trajectories seems to be in favor of the EPH model compared to the TTM fitted. Indeed, for all the sampled directions, the EPH model, especially in its constant $\alpha(\rho)$ formulation, follows the reference TDDFT curves quite closely, except for the Incommensurate 3 direction (Fig. 5(d)) where the energy lost during the collision (the electronic density peak) is overestimated. The low density regions and in most cases the high density regions seem to be well reproduced by the EPH model, and the density dependence of the energy lost to electrons globally well followed. It can be easily noticed visually on Fig. 5(b) and Fig. 5(c) that the constant MD-EPH performs better than the quadratic one. Table III actually indicates that for incommensurate directions, the constant EPH always performs better than the quadratic one.

According to Table III, the TTM fitted performs worse than the EPH constant case for all incommensurate directions, except for the incommensurate 3 case. The MAE difference is the greatest for the incommensurate 2 direction, with the $\mathrm{EPH}$ constant case having a MAE of $6.8 \mathrm{eV}$ and the TTM fitted a MAE of $29.2 \mathrm{eV}$. As in the channeling trajectories, the case in which the TTM fitted performs the worst is the case in which high electronic density values are sampled. The quadratic EPH reproduces the TDDFT data better than the TTM fitted for the incommensurate 1 (MAE of $9.3 \mathrm{eV}$ compared to $10.0 \mathrm{eV}$ ), the incommensurate $1+$ vacancy (MAE of $15.8 \mathrm{eV}$ compared to $18.4 \mathrm{eV}$ ), the incommensurate 2 (MAE of $14.5 \mathrm{eV}$ compared to $29.2 \mathrm{eV}$ ) directions, but worse for the incommensurate 3 (MAE of $26.1 \mathrm{eV}$ compared to $14.3 \mathrm{eV})$.

On the whole, the EPH constant and quadratic cases 
TABLE III. Distance (MAE) of the EPH and TTM fitted energy losses curves with respect to the reference TDDFT data.

\begin{tabular}{l|c|c|c} 
& EPH Constant $(\mathrm{eV})$ & EPH Quadratic $(\mathrm{eV})$ & TTM fitted $(\mathrm{eV})$ \\
\hline \hline Center channel $<001>$ & 9.4 & 12.9 & 18.8 \\
Center channel $<110>$ & 57.9 & 67.2 & 35.7 \\
Center channel $<111>$ & 5.4 & 3.4 & 18.7 \\
Off-center channel $<001>(1 / 2)$ & 4.2 & 13.4 & 3.6 \\
Off-center channel $<001>(1 / 4)$ & 15.5 & 8.0 & 32.0 \\
Off-center channel $<110>$ & 3.5 & 2.5 & 19.8 \\
Incommensurate 1 & 5.7 & 9.3 & 10.0 \\
Incommensurate 1 vacancy & 8.2 & 15.8 & 18.4 \\
Incommensurate 2 & 6.8 & 14.5 & 29.2 \\
Incommensurate 3 & 21.5 & 26.1 & 14.3 \\
\hline Total & 13.2 & 18.2 & 23.4
\end{tabular}

perform better than the TTM fitted, with the total MAE of the constant EPH case being of $13.2 \mathrm{eV}$, the one of the quadratic EPH of $18.2 \mathrm{eV}$ and the one of the TTM fitted of $23.4 \mathrm{eV}$. Visually, according to Fig. 4 and Fig. 5, the EPH model also allows to reproduce in many cases very closely the density dependence of the stopping observed in TDDFT simulations, which is, in addition to the quantitatively better MAE, more physically satisfying than the always constant stopping given by the TTM fitted.

Also, on the whole, the constant EPH performs better than the quadratic one (total MAE of $13.2 \mathrm{eV}$ compared to $18.2 \mathrm{eV}$ ). This can be surprising considering the relative complexity of the two functions, and the fact that an $\alpha(\rho)$ parameter shape similar in many ways to our quadratic expression was employed in [15]. This means that the density terms appearing as weighting factors in (3) are sufficient to guarantee a rich density dependent behavior of the stopping in the EPH model. An important reason for the less satisfactory performance of the quadratic EPH with respect to TDDFT data lies in the decay to zero we chose for the quadratic expression. Indeed, in light of the graphs of Fig. 4 and Fig. 5, this is questionable, as no step-like behavior of the stopping is observed in our TDDFT data as was the case in [15]. However, a decay is necessary otherwise the energy lost to electrons would diverge in regions of high electronic densities. As a compromise, it could be wise to set this decay to a non-zero value instead of strictly zero.

Regarding the constant parametrization of the EPH model, despite the good results it provides, the fact that $\alpha$ is non-zero when $\rho=0$ ought to be discussed. Indeed, as we said earlier, it is physically more satisfactory to have $\alpha(\rho) \approx 0$ at low $\rho$. A consequence of this might be an overestimation of the strength of the electron-phonon coupling since the part of the $\alpha(\rho)$ function that governs the magnitude of electron-phonon coupling is the low $\rho$ one. This is an aspect we can only evaluate by performing MD-EPH simulations of collision cascades with different thermal parameters of the EPH model, as we have done with the TTM in [13]. To provide an answer to this question, among other objectives, the next section is dedicated to simulations of collision cascades with the EPH model and with the TTM for comparison.

\section{MD SIMULATIONS OF COLLISION CASCADES}

\section{A. TTM and EPH parameters employed in the simulations}

In this section, simulations of collision cascades in $\mathrm{Si}$ with the TTM and the EPH model are carried out. With the EPH model, cascades with the constant and the quadratic parametrization of the parameter $\alpha(\rho)$ are performed. With the TTM, we only ran cascades using the SRIM derived parametrization of the TTM, as it is the default and most widely employed way to parametrize the TTM. Moreover, it is highly unlikely that TDDFT simulations will be used to parametrize the TTM for collision cascade simulations, as we did in the previous section. The comparison between the results of the cascades with the EPH model and with the TTM we perform in this section should thus be envisioned more as a comparison between realistic usages of the models, rather than a purely theoretical comparison between both models. If the electronic stopping parameter $\gamma_{s}$ of the TTM is obtained with SRIM, the electron-phonon coupling parameter $\gamma_{p}$ of the TTM is chosen on the basis of a careful literature search, which led us to choose the value of the electronphonon coupling presented in [39]. The electronic stopping parameter $\gamma_{s}$ obtained with SRIM in Si is equal to $39 \mathrm{~g} / \mathrm{mol} / \mathrm{ps}$ (LAMMPS metal units), which yields at $10 \mathrm{keV}$ an electronic stopping power of about $11 \mathrm{eV} / \AA$. The value of the electron-phonon coupling $\gamma_{p}$ that we use is $25 \mathrm{~g} / \mathrm{mol} / \mathrm{ps}$ (LAMMPS metal units). Considering that in the TTM the total friction coefficient acting on atoms is the sum of $\gamma_{s}$ and $\gamma_{p}$, both actually contribute to the stopping [8]. At $10 \mathrm{keV}$, the $\gamma_{p}$ parameter we use yields an electronic stopping of about $7 \mathrm{eV} / \AA$. In the following when we mention the effects of electron-phonon coupling in the TTM we do not refer to the effects of the $\gamma_{p}$ parameter as part of the total friction force (elec- 
tronic stopping effects), but to the energy fed back from the electrons to the ions via electron-phonon coupling, whose magnitude is proportional to $\gamma_{p}$. We recall that for the EPH model, no value of the electron-phonon coupling must be specified because both electronic stopping power and electron-phonon coupling are obtained from the $\alpha(\rho)$ parameter.

To evaluate the influence of the thermal parameters of the EPH model and of the TTM, i.e. the electronic specific heat $C_{e}$ and the electronic thermal conductivity $\kappa_{e}$, simulations with different values of these parameters are carried out. In all the simulations launched, $C_{e}$ and $\kappa_{e}$ are considered constant. We employ this approximation so that the effects of $C_{e}$ and $\kappa_{e}$ are easier to observe and discuss. In reality, $C_{e}$ and $\kappa_{e}$ depend on the electronic temperature $T_{e}$. Reliable $T_{e}$-dependent values at low $T_{e}$ for $\kappa_{e}$ can be found for Si in [40], and can be extended to $C_{e}$ with the relation $\kappa_{e}\left(T_{e}\right)=\rho D_{e} C_{e}\left(T_{e}\right)$. For practical purposes, for the calculation of $C_{e}$ and $\kappa_{e}$, the electronic diffusivity $D_{e}$ and the electronic density $\rho$ can be considered constant and respectively equal to $20000 \AA^{2} / \mathrm{ps}$ and $0.05 \mathrm{e}^{-} / \AA^{3}$ [41]. The procedure to obtain an expression for $C_{e}$ (and $\kappa_{e}$ consequently) for each possible $T_{e}$ is explained in [13].

Three scenarios are defined:

- Scenario 1 refers to a case where $C_{e}$ and $\kappa_{e}$ are deliberately set to low values compared to what could be considered as the reference electronic temperature $T_{e}$ dependent ones. In scenario $1, C_{e}=$ $5 \times 10^{-6} \mathrm{eV} / \mathrm{K}$ and $\kappa_{e}=5 \times 10^{-3} \mathrm{eV} / \mathrm{K} / \AA / \mathrm{ps}$. It corresponds to realistic values of $C_{e}$ and $\kappa_{e}$ at low $T_{e}$, but not on the entire range of $T_{e}$ spanned in the simulations [13].

- Scenario 2 refers to a case where $C_{e}$ and $\kappa_{e}$ are purposely set to high values compared to what could be considered as the reference electronic temperature $T_{e}$ dependent ones. In scenario $2, C_{e}=3 / 2 k_{B}=$ $1.29 \times 10^{-4} \mathrm{eV} / \mathrm{K}$ and $\kappa_{e}=1.29 \times 10^{-1} \mathrm{eV} / \mathrm{K} / \AA / \mathrm{ps}$. It corresponds to realistic values of $C_{e}$ and $\kappa_{e}$ at high $T_{e}$, but not in the entire range of $T_{e}$ spanned in the simulations [13].

- Scenario 3 refers to a mix between scenario 1 and scenario 2: $C_{e}$ takes the high value it has in scenario 1 and $\kappa_{e}$ the low value it has in scenario 2 . In scenario $3, C_{e}=1.29 \times 10^{-4} \mathrm{eV} / \mathrm{K}$ and $\kappa_{e}=5 \times$ $10^{-3} \mathrm{eV} / \mathrm{K} / \AA / \mathrm{ps}$.

These three scenarios are employed with the TTM and with the constant parametrization of the EPH model. Only scenario 3 is used with the EPH quadratic parametrization, as our results with the constant parametrization of the TTM show, as we will see later in this section, that it is not necessary to perform simulations with all the thermal parameters scenarios in the quadratic EPH case.

Electronic grids made of $15 \times 15 \times 15$ cubic cells are employed both with the EPH model and the TTM.

\section{B. Cascades properties and statistical quantities}

The results are analyzed in terms of number of defects (number of Frenkel pairs), number of clusters and PKA penetration depth. The defects are counted with the Lindemann sphere criterion [42] and a radius of $0.45 \AA$, which was demonstrated to yield for each cascade an almost constant ratio to the number of defects obtained with the Wigner-Seitz method [43]. The proportionality factor between the two methods that we find in $\mathrm{Si}$ is of 8 , the same value was found by Nordlund et al. in [43]. It is then possible to write, for $\mathrm{Si}, N_{\text {lin }} \approx 8 \times N_{w-s}$ where $N_{l i n}$ is the number of defects obtained with the Lindemann method and $N_{w-s}$ is the number of defects obtained with the Wigner-Seitz method. Two defects are considered to belong to the same cluster if they are separated by a distance smaller than twice the nearest neighbor distance, i.e. $4.7 \AA$ in Si. The PKA penetration depth is defined in [33].

To quantify the number of defects, the number of clusters and the PKA penetration depth, we use the mean values calculated over the 75 calculations carried out for each case studied, as well as graphical representations in the form of box plots. In the box plots are represented the minimum and maximum values of each set, as well as the first quartile, median (or second quartile) and third quartile values of each set. The uncertainties of the mean values are quantified with the calculations of the Standard Error of the Mean (SEM) quantities.

\section{Results and discussions}

Table IV summarizes the parameters employed in the various collision cascades scenarios we simulated, and gives the mean values of the number of defects, the number of clusters and the mean PKA penetration depth for each of these scenarios. Fig. 6 and Fig. 7 respectively show the distributions of the number of defects and of the PKA penetration depth as box plots for all the studied simulation scenarios.

\section{Defects and clusters evolution}

From Fig. 6, the similarity between all the EPH scenarios is immediately striking. The different thermal parameters employed in the constant case as well as the switch between quadratic or constant parametrizations do not lead to any visible effects on the number of defects. The values of the first, second and third quartiles are comparable between each studied scenario. The minimum and maximum values show larger discrepancies but this cannot be attributed to changes in the thermal parameters or in the $\alpha$ function employed. The high degree of stochasticity of the cascades makes it very difficult to obtain converged minimum and maximum values for a given set of simulations. Consequently, it is very hard to 
TABLE IV. Summary of the parameters employed in the different sets of cascades simulations carried out and mean values of the number of defects, the number of clusters and of the PKA penetration depth for all the simulation sets. The values in brackets next to the mean values are the SEM values. Each mean value is calculated from a set of 75 simulations initiated with distinct PKA directions. This table only gives a summary of the parameters employed, more details can be found in the text.

\begin{tabular}{l|c|c|c|c|c|c|c} 
Model employed & \multicolumn{5}{|c|}{ EPH } & \multicolumn{3}{c}{ TTM (SRIM) } \\
\hline$\alpha$ function (for EPH) & Quadratic & \multicolumn{3}{|c|}{ Constant } & 1 & 2 & 3 \\
Thermal scenario name & 3 & 1 & 2 & 3 & Low & High & High \\
$C_{e}$ & High & Low & High & High & Low & High & Low \\
$\kappa_{e}$ & Low & Low & High & Low & Low & SRIM + [39] \\
Elec. stopp. + el-ph & Fitted & Fitted & Fitted & Fitted & SRIM $+[39]$ & SRIM $+[39]$ & SRIM \\
\hline Mean number of defects & $767(21)$ & $730(17)$ & $763(21)$ & $719(16)$ & $1299(47)$ & $473(10)$ & $555(17)$ \\
Mean number of clusters & $46(1)$ & $47(1)$ & $46(1)$ & $46(1)$ & $21(1)$ & $38(1)$ & $33(1)$ \\
Mean PKA depth $(\AA)$ & $156(7)$ & $160(7)$ & $151(7)$ & $150(8)$ & $151(6)$ & $150(7)$ & $144(7)$
\end{tabular}

draw conclusions from the minimum and maximum values only. The similarities in terms of number of defects between all the scenarios studied with the EPH model can also be observed in the mean values displayed in Table IV, differing by up to 48 defects and having SEM values of about 20 each time. The mean number of clusters displayed in Table IV is even more similar, with mean values differing at most by one cluster only.

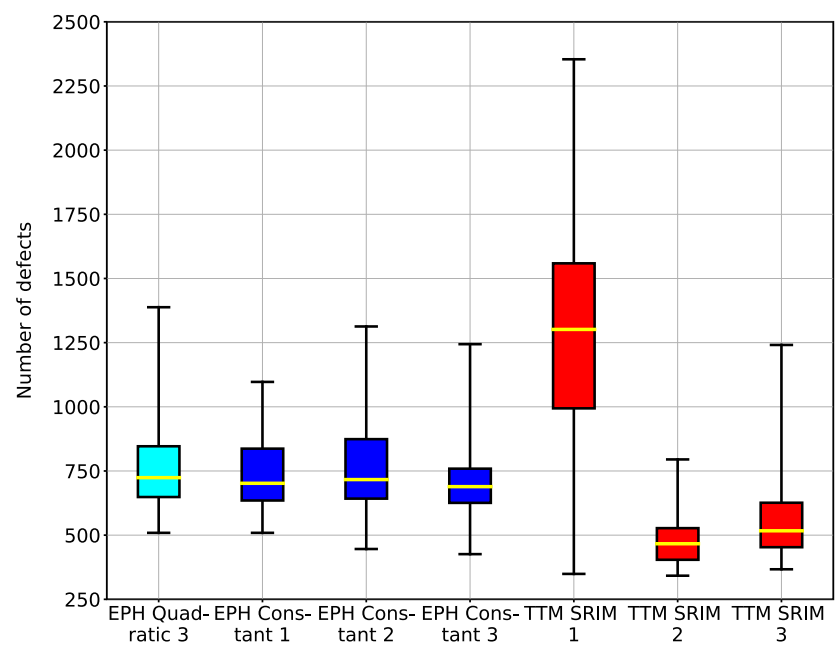

FIG. 6. Box plots of the number of defects for all the studied sets of parameters with the TTM and EPH model. From lowest to highest, the horizontal lines of a box plot represent the minimum value of the set, the first quartile value, the median (or second quartile value, in yellow), the third quartile and the maximum value of the set.

The fact that the constant and quadratic parametrizations of the EPH model give similar defects number is not surprising considering both parameters were fitted to reproduce the same reference TDDFT data. More interestingly and less expected, the fact that the different thermal scenarios used in the constant $\alpha$ case (which, as we said earlier, should overestimate the magnitude of the electron-phonon coupling), show no conclusive variations in the number of defects reveals that the electron-phonon coupling has no effect on the defects formation. Indeed, the thermal parameters $C_{e}$ and $\kappa_{e}$ of the EPH model (and of the TTM), do not act on the friction force acting on the moving projectile. They only control the temporal rate of the electronic energy lost $\left(C_{e}\right)$ to the ions and the diffusion of the electronic energy to nearby electrons $\left(\kappa_{e}\right)$. Thus, in Si at $10 \mathrm{keV}$, no defect is created due to the energy fed back from the electrons to the ions via the electron-phonon coupling. In the standard SRIM parametrization of the TTM, with the electron-phonon coupling parameter taken from [39], we found in [13] that electron-phonon coupling had an effect on the creation of defects in Si and Ge. In the above mentioned article, the simulations were carried out at the lower temperature of $100 \mathrm{~K}$. In this paper, we run new collision cascade simulations with the TTM with a thermostat temperature of $300 \mathrm{~K}$, as it is done for the EPH model. This allows us to directly compare the results of the EPH and TTM collision cascades.

By looking at the box plots of the TTM scenarios in Fig. 6, it can be observed that the distributions of the number of defects between the studied thermal scenarios show impressive discrepancies. The entire distribution of scenario 1 is obviously shifted upwards compared to scenarios 2 and 3. Quantitatively, the median values in scenarios 2 and 3 are respectively 467 and 517, while it is 1308 in scenario 1 . The first quartile values in scenarios 2 and 3 are respectively 404 and 453, while it is 994 in scenario 1 . The third quartile values in scenarios 2 and 3 are respectively 527 and 626 , while it is 1559 in scenario 1 . This trend is confirmed by the mean values of the number of defects of Table IV, which are similar for scenarios 2 and 3 (473 and 555 respectively) and about 3 times greater for scenario 1 (1383). This is in line with the parametric study of the TTM we performed in [13], where we observed that more defects were created with low values of $C_{e}$ and $\kappa_{e}$ (in the cited article, $\kappa_{e}=\rho D_{e} C_{e}$, with $D_{e}$ and $\rho$ constant). However, in the previous parametric study we performed on the TTM, the discrepancies between similar scenarios for the thermal parameters were much smaller. We checked the calculation of our number of defects with the Weigner-Seitz method of the OVITO software [44], and found the same factor of about 3 between the mean values. The fact that the present cal- 
culations are carried out at $300 \mathrm{~K}$ instead of $100 \mathrm{~K}$ like we did in [13] could explain these enhanced discrepancies. As clearly explained in [13] and above in this article, the only way the thermal parameters $C_{e}$ and $\kappa_{e}$ can influence the number of defects is via the electron-phonon coupling. If the energy fed back from the electrons to the ions via electron-phonon coupling is enough to induce the melting of the material, the number of observed defects increases. Carrying out simulations at $300 \mathrm{~K}$ may favor this melting behavior.

The comparison of the number of clusters also gives valuable information regarding the melting of the material in scenario 1 with the TTM. Indeed, according to Table IV, we find on average 38 and 33 clusters in the TTM scenarios 2 and 3, and only 21 for scenario 1 . Thus, with scenario 1 , more defects are contained in a smaller number of clusters, which means that the clusters are bigger. Looking at the size and shape of the clusters of defects, in the case of scenario 1 , for almost all the cascades, the defects are almost all contained in very large amorphous pockets, defined as clusters containing more than 100 defects. Those amorphous clusters are by far less important in cascades of scenarios 2 and 3: $76 \%$ of defects are contained in amorphous clusters in scenario 1 whereas only $10 \%$ and $25 \%$ in scenario 2 and 3 respectively. The low values of $C_{e}$ and $\kappa_{e}$ indeed induced melting via electron-phonon coupling, leading to these large amorphous pockets.

From the basic heat diffusion equation of the TTM [8], a low value of $C_{e}$ results in a high rate of energy exchange between electrons and ions [45], and a low value of $\kappa_{e}$ results in a poor distribution of electronic heat in the material. The electronic energy is contained in very specific regions of the material and is very quickly fed back to the ions of the same regions, thus inducing melting.

From the figures mentioned above, it also appears that a bit more defects are observed in scenario 3 compared to scenario 2, as well as less clusters. It also indicates an increase in melting of the material due to the low value of $\kappa_{e}$ employed in scenario 3. However, this melting is by far less important than in scenario 1 . It means that the combined effects of $C_{e}$ and $\kappa_{e}$ are responsible for the large melting observed in scenario 1 . Note that with the SW potential, the lattice thermal parameters of $\mathrm{Si}$ (melting point and lattice thermal conductivity) are well reproduced [46], the melting behavior of the material is therefore realistic.

The important point to remember from those MDTTM cascades calculations in $\mathrm{Si}$ is that with the TTM, electron-phonon coupling can have very large effects on the number of defects in collision cascades in $\mathrm{Si}$. On the contrary, with the EPH model, the electron-phonon coupling was not found to have any effect. Despite the fact the parameters chosen for $\kappa_{e}$ and $C_{e}$ in scenario 1 are unrealistic, this is a first clue pointing to the fact that the TTM in its original form [8], and parametrized with SRIM for $\gamma_{s}$ and a literature search for $\gamma_{p}$, might overestimate the effects of the electron-phonon coupling in collision cascades. Another argument that supports this point is that experimentally, amorphization of Si by electronic mechanisms occurs with swift irradiation of heavy ions of considerably greater energy than our $10-\mathrm{keV}$ collision cascades, i.e. tens of $\mathrm{MeV}[47,48]$. Thus, melting (amorphization) of the material via electron-phonon coupling as important as the one we observe with the TTM in scenario 1 is highly unrealistic. To counterbalance what we just said, one could argue that the overall lower electronic stopping power at stake in the EPH model compared to the TTM makes the amount of energy lost to electrons smaller than with the TTM and thus reduces the possibility to observe electron-phonon coupling effects because there is less energy to give back to the ions. MD-EPH simulations with various values of constant $\alpha$ and performed on a system where thermal effects are more obvious than in Si, like Ge for example $[13,46]$, could help to shed light on this specific point.

We now focus on scenarios 2 and 3 of the TTM and 1 , 2 and 3 for the EPH model, for which the energy transfer via electron-phonon coupling has very few effects on the number of defects. The visual comparison of the distributions of the number of defects shown in Fig. 6 with the EPH model and the TTM immediately reveals that many more defects are created with the EPH model than with the SRIM parametrization of the TTM. The median of the number of defects for all scenarios in the EPH model ranges from 719 and 763, whereas the TTM scenario 2 and 3 have a median of 467 and 517 . The mean values of Table IV display roughly the same discrepancy, with mean values ranging from 719 to 767 with the EPH model and mean values of 473 (scenario 2) and 555 (scenario 3) with the TTM in its SRIM parametrization. This can be easily explained by the fact that the amount of energy lost to the electrons obtained with the EPH model fitted to the reference TDDFT data is much lower than the amount of energy lost to the electrons with the SRIM parametrization of the TTM, as shown in Fig. 4 and Fig. 5. Consequently, as less energy is lost to electrons, more energy is available to create defects via collisions between ions or local melting of the matter as it occurs in semiconducting materials [43, 49]. This highlights the significance of the existing controversy between SRIM and TDDFT electronic stopping calculations, as we prove here it has an important impact on the number of defects created during collision cascades. To be complete on this point, note that in the TTM, the electronphonon coupling parameter $\gamma_{p}$ also contributes to the friction force, the total friction coefficient being the sum of the electronic stopping parameter $\gamma_{s}$ and $\gamma_{p}$. The discrepancies between the number of defects observed with the EPH and the TTM scenarios are therefore enhanced by this aspect of the TTM.

To conclude on the defects analysis, the results obtained with the EPH model and with the TTM would be much more similar if we had employed the TTM parameters we fitted to the TDDFT data in Section IV. However, a TTM parametrization with TDDFT data is 
not representative of how the TTM is used in the literature. Here, we wanted to compare the results obtained following the default guidelines for the parametrization of both the EPH model [15] and the TTM [8].

\section{PKA penetration depth}

The analysis of the PKA penetration depth is more straightforward than the number of defects and clusters. The mean values of Table IV reveal that the mean PKA penetration depth for the EPH scenarios are all comprised between $150 \AA$ and $160 \AA$. The SEM values (about 8) accompanying these mean values lead to consider the PKA penetration depth in all EPH scenarios as similar: no evolution or trends in the mean PKA depth can be observed. The fact that the thermal scenarios of the constant parametrization of the EPH model do not give different values of the PKA penetration depth is expected, as both $C_{e}$ and $\kappa_{e}$ do not act on the friction force to which the moving ions are subjected. Considering the similarities in the electronic stopping between the constant and quadratic parametrization of the EPH model for all directions studied in Section IV, the very similar mean values of the PKA penetration depth for the $\mathrm{EPH}$ constant and quadratic cases are also consistent.

The mean values of the PKA penetration depth for the TTM scenarios are also very consistent (151 A, $150 \AA$ and $144 \AA$ for scenarios 1, 2 and 3 respectively), for the same reasons as just exposed for the EPH thermal scenarios. However, it seems that the mean values of the PKA penetration depth of the EPH model are slightly greater than the ones obtained with the SRIM parametrization of the TTM. The uncertainties (SEM values) coming with those mean values do not allow to be completely conclusive on this point. The analysis of the box plots of Fig. 7 do not give much more information on this aspect as all the distributions seem to be quite similar: no clear trend or behavior can be observed.

An increase in the PKA penetration depth with the EPH model would be physically sound. Indeed, the electronic stopping with the EPH model is overall smaller than with the SRIM parametrization of the TTM we adopted. Moreover, with the EPH model, the electronic stopping is specifically low for channelling directions (see Fig. 4), allowing for an increased depth of the PKAs if they are themselves in a channelling direction. The slight increase in the mean PKA penetration depth for the EPH model is consistent with this, even if it is less blatant than one might expect. If it is hard to be conclusive about the impact of the electronic stopping on the PKA depth with our simulations, it is obvious that the electronic stopping can have an important impact on the creation of defects, especially in semiconducting materials where the formation of amorphous pockets of defects can be observed.

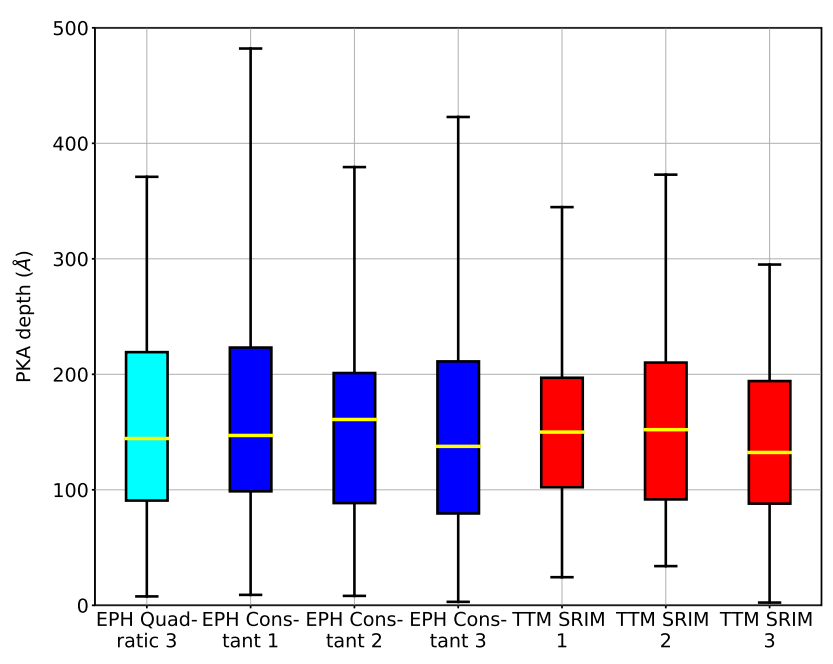

FIG. 7. Box plots of the PKA penetration depth for all the studied sets of parameters with the TTM and EPH model. From lowest to highest, the horizontal lines of a box plot represent the minimum value of the set, the first quartile value, the median (or second quartile value, in yellow), the third quartile and the maximum value of the set.

\section{CONCLUSION}

First principles TDDFT calculations of electronic stopping were combined with the EPH model for the inclusion of non-adiabatic electronic effects into MD simulations of collision cascades. A simple parametrization of the EPH model with a constant $\alpha$ function already gives very satisfactory results in terms of electronic density (or crystal direction) dependence of the electronic stopping obtained with MD-EPH simulations in Si, considering TDDFT results as the reference. The TTM in its SRIM parametrization obviously does not allow reproduction of the TDDFT data. However, when parameters of the TTM are fitted to the TDDFT data, although the energy lost to electrons is independent of the electronic density and the friction parameter is scalar, the results obtained do not differ as much as one could expect from the TDDFT data. Nonetheless, the EPH model allows to better reproduce the electronic stopping calculated with TDDFT in Si than the TTM, whose incorporation of electronic stopping is not tensorial and density-dependent as in the EPH model. Indeed, quantitatively the agreement between the EPH model and TDDFT is better, and qualitatively it is more satisfactory physically for the electronic stopping to be dependent on the electronic density. By refining the $\alpha(\rho)$ expressions employed for the fitting, we believe even better reproduction of the TDDFT data can be achieved with the EPH model.

Collision cascades carried out in $\mathrm{Si}$ at $10 \mathrm{keV}$ have revealed that the choice of the model employed for the inclusion of the electronic effects and the parametrization 
of the chosen model have a significant influence on the number of defects created. Our collision cascade simulations performed with the EPH model fitted on TDDFT data give significantly more defects than cascades carried out with the TTM parametrized with SRIM calculations. Fundamental discussions comparing SRIM and TDDFT electronic stopping calculations are then of prime importance as it has visible effects on collision cascades even at the relatively low energy of $10 \mathrm{keV}$. The impact of the electron-phonon coupling on the creation of defects is found to be significant with the TTM and highly dependent on the $C_{e}$ and $\kappa_{e}$ parameters chosen, whereas no influence of the electron-phonon coupling on the creation of defects was observed with the EPH model in Si. This last aspect indicates a likely overestimation of the significance of electron-phonon coupling in the naive but widespread parametrization method we employed for the TTM. Additional calculations with the EPH model at different energies, with different parameters and on different systems would considerably help to be fully conclusive on the significance of electron-phonon coupling in collision cascades.

We believe this paper gives strong evidence in favor of the necessity for further studies comparing SRIM-derived and TDDFT-derived electronic stopping (actually on the understanding and quantification of electronic stopping in general). In particular, the issue of the charge state of the projectile, which was not addressed in this article, should be addressed in the future as it may have significant quantitative implications on the stoppings cal- culated with TDDFT. In addition, this paper provides trustworthy arguments defending the significant impact the finer incorporation of electron-phonon coupling in the EPH model has on the results of collision cascades.

\section{ACKNOWLEDGMENTS}

The authors would like to thank Andre Schleife and Cheng-Wei Lee from University of Illinois UrbanaChampaign as well as Alfredo Correa and Artur Tamm from Lawrence Livermore National Laboratory for fruitful discussions. The authors would also like to thank Chloé Simha for proofreading this manuscript. This article is based upon work from COST Action TUMIEE CA17126, supported by COST (European Cooperation in Science and Technology). Calculations have been performed using HPC resources from GENCI-CCRT supercomputer at $\mathrm{CEA}, \mathrm{DAM}$, DIF, HPC resources from GENCI (Grant A0030907474) and HPC resources from CALMIP (Grant 1555). J. Teunissen and F. Da Pieve have received funding from the Research Executive Agency under the EU's Horizon2020 Research and Innovation program, project ESC2RAD (grant ID 776410). T. Jarrin, A. Hémeryck and N. Richard are active members of the Multiscale and Multi-Model Approach for Materials in Applied Science consortium (MAMMASMIAS consortium), and acknowledge the efforts of the consortium in fostering scientific collaboration.
[1] M. Yamaguchi, Sol. Energy Mater. Sol. Cellule 68, 31 (2001).

[2] B. Milbrath, A. Peurrung, M. Bliss, and W. Weber, Journal of Materials Research 23, 2561-2581 (2008).

[3] J. Lindhard, M. Sharff, and H. Schiott, Matisk-fysiske Meddelelser det Kongelige Danske Videnskabernes Selskab 33, 1 (1963).

[4] J. Lindhard and W. A, Matisk-fysiske Meddelelser det Kongelige Danske Videnskabernes Selskab 34, 1 (1963).

[5] G. Moliere, Zeitschrift für Naturforschung A 2, 133 (1947).

[6] J. Ziegler, J. Biersack, and U. Littmark, The Stopping Range of Ions in Solids, 1st ed. (Pergamon Press, N.Y, 1983).

[7] Y. Zhong, K. Nordlund, M. Ghaly, and R. S. Averback, Phys. Rev. B 58, 2361 (1998).

[8] D. Duffy and A. Rutherford, J. Phys.: Cond. Matter. 19, 1 (2007).

[9] A. Caro and M. Victoria, Phys. Rev. A 40, 2287 (1989).

[10] G. E. Uhlenbeck and L. S. Ornstein, Phys. Rev. 36, 823 (1930).

[11] S. Lohmann, R. Holeňák, and D. Primetzhofer, Phys. Rev. A 102, 062803 (2020).

[12] C.-W. Lee, J. A. Stewart, R. Dingreville, S. M. Foiles, and A. Schleife, Phys. Rev. B 102, 024107 (2020).

[13] T. Jarrin, A. Jay, A. Hémeryck, and N. Richard, accepted for publication in Nucl. Instr. Meth. Phys. R. B
(2020).

[14] A. Tamm, M. Caro, A. Caro, G. Samolyuk, M. Klintenberg, and A. A. Correa, Phys. Rev. Lett. 120, 185501 (2018).

[15] M. Caro, A. Tamm, A. A. Correa, and A. Caro, Phys. Rev. B 99, 174301 (2019).

[16] R. Ullah, E. Artacho, and A. A. Correa, Phys. Rev. Lett. 121, 116401 (2018).

[17] A. Caro, A. A. Correa, A. Tamm, G. D. Samolyuk, and G. M. Stocks, Phys. Rev. B 92, 144309 (2015).

[18] A. Tamm, M. Caro, A. Caro, and A. A. Correa, Phys. Rev. B 99, 174302 (2019).

[19] For documentation of the USER-EPH fix implemented for LAMMPS: https://github.com/LLNL/USER-EPH.

[20] R. Kubo, Rep. Prog. Phys. 29, 255 (1966).

[21] I. Koponen, Phys. Rev. B 47, 14011 (1993).

[22] A. Tamm, G. Samolyuk, A. A. Correa, M. Klintenberg, A. Aabloo, and A. Caro, Phys. Rev. B 94, 024305 (2016).

[23] J. Yang, http://opium.sourceforge.net (2018).

[24] F. Gygi, IBM Jour. Res. Dev. 52, 137 (2008).

[25] E. Draeger, X. Andrade, J. Gunnels, A. Bhatele, A. Schleife, and A. Correa, J. Par. Dist. Comp. 106, 205 (2017).

[26] A. A. Correa, Comp. Mat. Sci. 150, 291 (2018).

[27] C. Kittel, Introduction to Solid State Physics, 8th ed. (John Wiley and Sons, 2004). 
[28] D. C. Yost, Y. Yao, and Y. Kanai, Phys. Rev. B 96, 115134 (2017).

[29] A. Schleife, Y. Kanai, and A. A. Correa, Phys. Rev. B 91, 014306 (2015).

[30] S. Plimton, J. Comput. Phys. 117, 1 (1995).

[31] F. H. Stillinger and T. A. Weber, Phys. Rev. B 31, 5262 (1985).

[32] N. Chen, E. Rasch, D. Huang, E. Heller, and F. Gao, IEEE Trans. Nucl. Sc. 65, 1108 (2018).

[33] T. Jarrin, A. Jay, M. Raine, N. Mousseau, A. Hémeryck, and N. Richard, IEEE Trans. Nucl. Sc. 67, 1273 (2020).

[34] T. Jarrin, A. Jay, N. Richard, and A. Hémeryck, Nucl. Instr. Meth. Phys. R. B 500-501, 1 (2021).

[35] J. Ziegler, M. Ziegler, and J. Biersack, Nucl. Instr. Meth. Phys. R. B 268, 1818 (2010).

[36] A. Lim, W. M. C. Foulkes, A. P. Horsfield, D. R. Mason, A. Schleife, E. W. Draeger, and A. A. Correa, Phys. Rev. Lett. 116, 043201 (2016).

[37] M. Caro, A. Tamm, A. Correa, and A. Caro, J. Nucl. Mat. 507, 258 (2018).

[38] P. Giannozzi et al., J. Phys.: Cond. Matter. 21, 395502 (2009).

[39] A. Chettah, H. Kucal, Z. Wang, M. Kac, A. Meftah, and M. Toulemonde, Nucl. Instr. Meth. Phys. R. B 267, 2719 (2009).

[40] C. Glassbrenner and G. Slack, Phys. Rev. A 134, 1058 (1966).
[41] M. Toulemonde, C. Dufour, A. Meftah, and E. Paumier, Nucl. Instr. Meth. Phys. R. B 166-167, 903 (2000).

[42] H. Hensel and H. M. Urbassek, Phys. Rev. B 57, 4756 (1998).

[43] K. Nordlund, M. Ghaly, R. S. Averback, M. Caturla, T. Diaz de la Rubia, and J. Tarus, Phys. Rev. B 57, 7556 (1998).

[44] A. Stukowski, Mod. Sim. Mat. Sci. Eng. 18, 015012 (2010).

[45] G. Khara, S. T. Murphy, S. L. Daraszewicz, and D. M. Duffy, J. Phys.: Cond. Matter. 28, 395201 (2016).

[46] P. Lopez, L. Pelaz, I. Santos, L. Marques, and M. Aboy, J. App. Phys. 111, 033519 (2012).

[47] A. Kamarou, W. Wesch, E. Wendler, A. Undisz, and M. Rettenmayr, Phys. Rev. B 78, 054111 (2008).

[48] H. Amekura, M. Toulemonde, K. Narumi, R. Li, A. Chiba, Y. Hirano, K. Yamada, S. Yamamoto, N. Ishikawa, N. Okubo, and Y. Saitoh, Sci. Rep. 11, 185 (2021).

[49] K. Nordlund, S. Zinkle, S. A.E, F. Granberg, A. R.S, R. Stoller, T. Suzudo, L. Malerba, F. Banhart, W. Weber, F. Willaime, S. Dudarev, and D. Simeone, J. Nucl. Mat. 512, 450 (2018). 